\title{
Small telescopes may be auctioned off under US 'privatization' plan
}

Washington. Most of the small telescopes at present available to all US astronomers would be closed or sold to private operators under a restructuring proposed for the National Optical Astronomy Observatories (NOAO). NOAO, which includes Kitt Peak National Observatory in Arizona and the Cerro Tololo Inter-American Observatory in Chile, hopes to compensate for the loss by building three new instruments for night-time observing. These would be a 2.4-metre telescope for each hemisphere, and a 4-metre telescope in Chile, built in cooperation with the University of North Carolina and with Brazil.

Another instrument for solar observations would be built at Kitt Peak and Sacramento Peak in New Mexico. The Association of Universities for Research in Astronomy (AURA), which runs NOAO, last month asked the National Science Foundation for $\$ 21.6$ million to

Faced with shrinking astronomy funding from the National Science Foundation, NOAO says it has no choice but to close or transfer eight of its smaller, older telescopes in Arizona and Chile. The only instruments that would remain in operation are the 4metre Mayall and 3.5-metre WIYN telescopes on Kitt Peak and the 4-metre Blanco telescope (and perhaps a 1.5-metre infrared telescope) at Cerro Tololo. In addition to the three new telescopes to be completed by 2001, NOAO users would have access to the twin 8-metre Gemini telescopes, the first due to begin operating in 2000 .

The proposals follow a recommendation by the National Research Council (NRC) last year that NOAO should place top priority on building advanced new telescopes, and keep the older telescopes open only if cover the new construction work.

Scanning the skies: Kitt Peak National Observatory would gain a telescope to compensate for closures.

\section{New funds bring Magellan project into focus}

Washington. The \$70-million Magellan project at Las Campanas in Chile is now officially a 'two-telescope' project, following a promise announced last week of funds from two more universities that will join the project's three existing backers.

The University of Michigan and the Massachusetts Institute of Technology (MIT) are joining the Carnegie Institution in Washington, the University of Arizona and Harvard University to make up an expanded five-member consortium. Telescope observation time will be proportional to an institution's investment in capital and operating costs. Carnegie, which owns the additional money is available (see Nature 373, 179; 1995). But NSF's astronomy division has told NOAO to plan for level funding at best, and so the restructuring plan is designed to trim $\$ 1.35$ million a year from an annual operating budget of $\$ 27$ million.

\section{IMAGE UNAVAILABLE FOR COPYRIGHT REASONS}

Further savings might be made if more partners buy into the proposed new telescopes.

Sidney Wolff, the director of NOAO, admits that closing telescopes means fewer observers. But the addition of three new telescopes would mean that "a large fraction of current users still would be served," says John Salzer, an astronomer from Wesleyan University, Middletown, Connecticut, who chairs NOAO's user committee. The practice of 'queue scheduling', under which NOAO staff conduct observations rather than having astronomers visit the mountain themselves, would be one way of increasing the number of users.

But advanced technology and streamlined operations can go only so far. "Obviously, if you're going to close four telescopes and replace them with one, there's no way in the world you can get four times the science throughput," says Salzer.

NOAO observatories are used by nearly 1,000 researchers a year. Half of all US astronomers rely on them exclusively, as these are the only telescopes open to the entire community. Private observatories, although more numerous and in many cases

more capable, are open only to astronomers whose institutions pay for the facility. Furthermore, says Salzer, "the majority of NOAO users are [users of] smaller telescopes".

The restructuring means that many of these astronomers will have to fend for themselves. One option is for smaller institutions to pool their money to

buy time at larger observatories. Salzer's own university, Wesleyan, for example, has joined several others to buy time from Case Western Reserve University of Cleveland, Ohio, which owns one of the Kitt Peak telescopes.

Wolff expects that such consortia will eventually purchase the NOAO telescopes. Two previously closed telescopes on Kitt Peak have already been sold, and negotiations are under way for the sale of a third.

But Wolff warns that the "downside" to privatization is that "you do not have open competitive access for the community as a whole. We're moving to more and more restricted access to facilities, and that's a fundamental policy issue that the NSF really needs to consider."

The NSF is aware of the problem, says Hugh Van Horn, director of its astronomy division, and is considering the idea of allowing public access to private telescopes in exchange for NSF financing of advanced instrumentation - a key recommendation of last year's NRC report. "I regard this as one of the highest priority items" for NSF support of astronomy, says Van Horn. But this would apply only to the large telescopes now under construction, and is still far from sure, given NSF's funding uncertainties.

Even though the smaller NOAO telepresent Las Campanas site, will be entitled to 50 per cent of observation time and will remain the project's 'lead partner'.

The other institutions will each be entitled to 10 per cent of the observing time, the remaining 10 per cent reserved for Chilean astronomers free of charge. A completion date for the telescopes has not been announced. But the primary mirror for Magellan 1 has been cast and is "ready for grinding and polishing" and scheduled for installation around mid-1998.

The telescopes will be situated at an elevation of 2,400 metres, far from any city lights and surrounded by stable air. scopes are still very much in demand, Salzer agrees that building new, advanced instruments should have priority. "No matter how you cut it, the telescopes at Kitt Peak and [Cerro Tololo] are old, and they're not going to be viable telescopes for much longer." NOAO has found that it would be cheaper to build new telescopes than to upgrade the old ones to meet modern performance standards. In such circumstances, Salzer claims that the NOAO restructuring proposal with its promise of three new telescopes is probably the best option in an era of downsizing.

Tony Reichhardt 